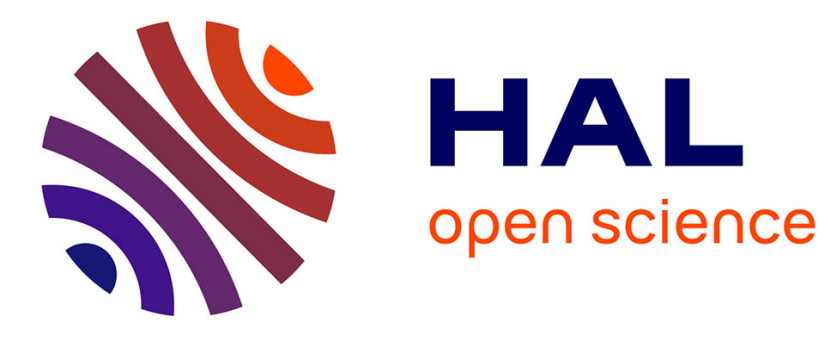

\title{
Style et intensité dans la prose de Mac Orlan \\ Stéphane Chaudier
}

\section{To cite this version:}

Stéphane Chaudier. Style et intensité dans la prose de Mac Orlan . Roman 20-50: Revue d'étude du roman du XXe siècle, 2009, Pierre Mac Orlan (Philippe Blondeau, dir.), nº 47, p. 79-90. hal-01675000

\section{HAL Id: hal-01675000 https://hal.science/hal-01675000}

Submitted on 3 Jan 2018

HAL is a multi-disciplinary open access archive for the deposit and dissemination of scientific research documents, whether they are published or not. The documents may come from teaching and research institutions in France or abroad, or from public or private research centers.
L'archive ouverte pluridisciplinaire HAL, est destinée au dépôt et à la diffusion de documents scientifiques de niveau recherche, publiés ou non, émanant des établissements d'enseignement et de recherche français ou étrangers, des laboratoires publics ou privés. 


\section{Stéphane Chaudier}

Publication : "Style et intensité dans la prose de Mac Orlan ", Pierre Mac Orlan, Roman 20-50 $\mathrm{n}^{\circ}$ 47, études réunies par Philippe Blondeau, juin 2009, p. 79-90.

Auteur : Stéphane Chaudier, université Lille, laboratoire ALITHILA, EA 1061

Mots-clés : Mac Orlan, Le Quai des brumes, Le Bal du Pont du Nord, style, hyperbole, comparaison, subjectivité

\section{Résumé :}

Dans l'univers littéraire de Mac Orlan, il semble que la sensation qui importe, celle qui est digne d'être notée, soit celle qui se signale par son caractère "intense" - révélant ainsi l'excès comme la dimension constitutive de la vie : "il reniflait le feu, se rôtissait les jambes, le plus qu'il pouvait" (nous soulignons). L'intensité semble caractériser aussi bien la chose que le sujet sensible qui l'accueille ou se porte audevant d'elle, selon un mouvement qui alterne ou combine élan et réceptivité. On verra tout d'abord comment cette écriture de l'intensité évite les pièges d'une rhétorique de l'hyperbole ; comment elle exploite ou déjoue le stéréotype là-même où il est le plus inévitable, dans l'expression du haut degré. Mais cette écriture des intensités ne vise-t-elle que l'expression du simple plaisir d'être vivant ? Si l'intensité constitue le ressort épique, tout autant que lyrique de cette écriture, celle-ci n'ignore pourtant pas la baisse d'intensité, ce qu'on appelle communément l'ennui. Cette approche de la vie comme intensité fait alors surgir une figure problématique de héros : le personnage de Mac Orlan est tour à tour fécondé et déserté par l'intensité, et jamais sûr d'entrer dans un rapport juste avec les multiples avatars de l'intensité.

\section{Style et intensité dans la prose de Mac Orlan}

«Un éclair... puis la nuit ! ». Plus encore que le jour, l'éclair s'oppose à la nuit ; car l'éclair, en se montrant, illumine tout autour de lui, alors qu'on ne voit pas le jour, même si c'est en lui qu'on voit toute chose. L'éclair est une lumière auréolée de sa propre visibilité ; l'éclair entre donc avec la lumière dans un rapport d'intensité. Tout objet qui apparaît à nos yeux glisse, porté par le temps, jusqu'au moment de sa disparition ; dans ce flux, le sujet sensible isole un instant : celui où il croit saisir l'objet à son plus haut degré de manifestation, - dans la plénitude de son être. On touche alors à la chose même, à ce qui importe. L'intensité appartient à l'objet car il n'est pas d'intensité qui ne soit intensité de rien ; mais l'intensité appartient à la conscience percevante, car il n'est pas non plus d'intensité sans une activité qui la saisisse comme telle. Affectant le sujet et l'objet, l'expérience de l'intensité définit un régime de perception que Lucien Jerphagnon nous aide à comprendre en opposant deux modalités du discours :

Il faut bien le reconnaître, on parle le plus souvent pour ne rien dire, ou dire des riens. [...] Il arrive cependant qu'on ait quelque chose à dire, à quoi l'on tient. [...] Dans toutes ces situations [...], une donnée demeure identique : c'est le même moi qui s'exprime, seul et unique à être soi à ce moment précis du temps $[\ldots]^{1}$.

Ce qui vaut pour le langage vaut pour la perception. La plupart du temps, nous sentons et ressentons de manière machinale ; mais parfois - et ce sont là des expériences d'intensité - la perception nous requiert tout entier : nous voyons mieux; simultanément, nous prenons conscience que nous voyons, et nous avons l'impression d'être enfin nous-mêmes. Dans un

\footnotetext{
${ }^{1}$ Lucien Jerphagnon, Au bonheur des sages, Paris, Desclée de Brower, 2004, repris en poche, coll. « Pluriel » (Hachette), pp. 9-10.
} 
même mouvement, nous éprouvons davantage - et nous-mêmes, et le monde. Je définirais volontiers les personnages de Marc Orlan, dans Le Quai des brumes et Le Bal du Pont du Nord ${ }^{2}$ comme des êtres voués à la quête de l'intensité.

Qu'un peintre, comme Michel Kraus, dise : "Toutes les forces aiment les pointes " ", cela n'a rien d'étonnant : la maxime est belle, mais elle est attendue ; après tout, c'est un artiste qui parle. Qu'un boucher, assassin minable de surcroît, s'exprime ainsi, et nous voilà face à une caractéristique non de l'esthète, mais de l'homme selon Mac Orlan :

J'aime particulièrement la musique religieuse. Ça m'élève l'âme et ça me pousse à toutes les extrémités. Nous avons tous une petite idée derrière la tête, tous sans exception ; cette idée, nous la connaissons mal nous-mêmes, elle est comme un veau mort-né, un fœetus, un poulet à ses débuts dans l'œuf. Il faut éviter de trouver à ses dépens le produit qui donne à cette idée une vie normale et puissante, une puissance plus forte que celle qu'un bœuf possède dans sa tête et dans son cou'.

Réfléchir sur ce qu'on ressent n'est pas l'apanage de l'artiste ; tous les personnages de Mac Orlan sont un peu les théoriciens de leur propre passion. Isabel, le boucher, se méfie des intensités ; il sait bien à quels excès elles portent. Mais c'est à Nelly, la prostituée, que revient le mérite de nouer de façon claire les notions d'intensité et de vie : " "la musique est neuve et neuf est tout ce qui me fait vivre" $"$. Comme tous les autres personnages, Nelly ne vit que portée par l'énergie qui « fait vivre », et qui est proprement l'intensité.

Peut-on définir la vie telle que l'expérience de l'intensité invite à la vivre ? Jean-Marie Ernst s'y essaie : il « regarda la vie se dérouler devant lui comme un film lointain, une chose animée, mais cependant morte et qui ne le touchait en rien $^{6} »$. La succession des connecteurs ( " mais », " cependant », " et ») signale le travail de la pensée. Elle engendre une distinction décisive, mais paradoxale : une chose a beau être animée, si elle ne me touche « en rien », elle est comme morte. La vie s'appréhende en termes relationnels : on est vivant, et le monde l'est tout autant que soi, quand on s'éprouve affecté par les réalités environnantes. Comment, en effet, ces réalités ne seraient-elles pas vivantes puisque ce sont elles qui nous apportent la conscience d'être vivant? La vie n'est pas le privilège de l'humain ; mais lui seul sans doute verbalise cette conscience : " d'être vivant doit être considéré comme une récompense, car la vie est une récompense offerte aux hommes pour on ne sait quelles qualités transcendantales ${ }^{7} »$, explique Mac Orlan. La vie (« le fait d'être vivant ») précède l'injonction (« doit être considéré ») qui elle-même précède la définition que l'écrivain en donne ( " car la vie est »). L'idée de "récompense » se suffit à elle-même : nul besoin de savoir de qui ou pourquoi on tient ce trésor. Nul besoin, non plus, de donner à ce participe passif, " offerte aux hommes », un agent qui serait Dieu. L'homme bénéficie d'un cadeau, et de cette récompense, il n'est redevable qu'à lui-même, qu'à ses propres qualités. Présentée avec la désinvolture qui sied à un romancier, cette philosophie est celle de Mac Orlan, - et elle nourrit son œuvre.

L'intensité est donc définie à la fois comme la manifestation et la conscience de la vie. Est intense tout moment au cours duquel le héros s'éprouve vivant dans un monde vivant. C'est pourquoi je souhaiterais étudier le langage de l'intensité, les formes variées par lesquelles le texte signifie et fait partager une telle expérience. Le langage de l'intensité décrit d'abord un contact : entre un fragment du monde et un personnage, une rencontre se fait dont le langage témoigne. Mais le rôle des mots ne s'arrête pas là ; ils examinent le devenir de chacune des

\footnotetext{
${ }^{2}$ Mac Orlan, Le Quai des brumes, Paris, Gallimard, 1927 ; repris en folio ; Le Bal du Pont du Nord, suivi de Entre deux jours, texte présenté par F. Lacassin, Paris, Gallimard, 1950, repris en folio. Nous utilisons désormais deux abréviations commodes : $Q B$ et $B P N$, suivies du numéro de la page de l'édition folio.

${ }^{3} Q B, 31$.

${ }^{4} Q B, 68$.

${ }^{5} Q B, 148$

${ }^{6} Q B, 96$.

${ }^{7} B P N, 17$.
} 
forces en présence : les unes dominent, les autres subissent. Mac Orlan ne lasse pas de raconter les péripéties de ce conflit permanent. C'est là le ressort épique de cette écriture, qui n'ignore pourtant pas la baisse d'intensité, ce qu'on appelle communément l'ennui. Cette approche de la vie comme intensité fait alors surgir une figure problématique de héros : le personnage de Mac Orlan est tour à tour fécondé et déserté par l'intensité, et jamais sûr d'entrer dans un rapport juste avec les multiples avatars de l'intensité.

\section{I- L'expression de l'intensité et ses enjeux}

Commençons, comme il se doit, par une construction dite « intensive », la corrélation consécutive introduite par le morphème «si » :

Son plaisir était de vivre une vie qu'elle inventait la nuit au «Lapin » devant quelques compagnons. Vie si éphémère qu'il suffisait d'un verre d'eau mêlé de rhum pour qu'elle s'évanouît dans la mémoire de la jeune fille ${ }^{8}$.

Sitôt qu'elle s'exprime, l'intensité, et c'est là un trait qui la caractérise, suscite des tensions qui font réfléchir. Se raconter des histoires est une expérience banale ; mais aspirée dans le champ de l'intensité, cette expérience devient problématique. Le groupe complexe « une vie qu'elle inventait la nuit au "Lapin" devant quelques compagnons » est repris par un simple nom, sans détermination, et qui le résume : "vie ». Cette simplification drastique des données de l'expérience fait surgir la tension : peut-on encore appeler «vie » des existences à ce point éphémères ? Non, sans doute ; et pourtant, pour Nelly, ces vies imaginaires sont de vraies vies, parce qu'elles lui sont données par et dans le plaisir : " son plaisir était de vivre une vie qu'elle inventait la nuit ». Le lecteur est alors invité à suspendre son jugement critique, à pénétrer comme par effraction - dans une intensité qui singularise le personnage ; une modalité insoupçonnée du " vivre » se révèle. Relisons : l'isolexisme " vivre une vie », la récurrence du son /i/, l'allitération en /v/, le choix qui consiste à présenter le procès selon l'effet intime qu'il produit sur celui qui l'éprouve (« vivre ») et non selon l'apparence qu'il revêt (« invent[er] » une histoire), tout ceci manifeste une intensité vécue. Son expression ne se limite pas à un mot, à une construction. "Éphémère » est intensif par rapport à « bref », et surtout si on rapporte cet adjectif au nom «vie » dont le signifié implique une certaine durée. Mais les syntagmes « son plaisir » et " vivre sa vie » participent aussi à cette " constellation » intensive qui, telle une traînée de feu, embrase toute la phrase. Le langage parvient à dire ce qui, profondément, compte.

Tous les procédés de l'intensité ne sont pas aussi subtils. Mac Orlan n'est pas avare de ces adjectifs hyperboliques qui sont autant de coups de cymbales ponctuant la phrase ; ils font résonner la matière romanesque à peu de frais : « il se glissait avec un plaisir indescriptible dans les draps ${ }^{9} »$; « des matelots passaient à côté d'elle dans l'obscurité indescriptible ${ }^{10}$ »; « Seppen écoutait le bruit surnaturel de la bataille ${ }^{11} »$; « Rabe avait tout d'un coup éprouvé une commisération infinie pour cette enfant ${ }^{12} »$. Dans Le Bal du Pont du Nord, l'adjectif « extraordinaire » revient comme une litanie. Il est question de « l'extraordinaire apparition de la flotte de sir Roger Keyes ${ }^{13}$ »; de « cette extraordinaire et furieuse mêlée ${ }^{14}$ ». La contagion gagne le discours des personnages : " "ce que vous dites là me fait penser à quelque chose d'extraordinaire, à quelque chose, Monsieur Pieter, qui devient extraordinaire"15 »; " "ces

\footnotetext{
${ }^{8} Q B, 59$.

$9 Q B, 8$.

${ }^{10} B P N, 179$

${ }^{11} B P N, 104$.

${ }_{12} Q B, 9$.

${ }^{13} B P N, 134$.

${ }^{14} B P N, 152$.

${ }^{15} B P N, 135$.
} 
ressemblances extraordinaires ne sont pas rares"16"; “"c'est pour moi une impression extraordinaire, je vous le jure, d'aller allumer ma pipe à l'endroit même où quelques années auparavant j'ai vécu une des nuits les plus épouvantables de la guerre" ${ }^{\not 17}$ ». Le relevé, qui isole ces segments, exagère sans doute le procédé ; mais il n'en reste pas moins sensible ${ }^{18}$.

Il ne s'agit pourtant pas de dénoncer un défaut du style. Répétées, ces épithètes à la fois banales et sonores indiquent le climat moral dans lequel baignent les personnages. Dans $\mathrm{Le}$ Quai des brumes, la suresthésie est l'effet de la misère :

Mais Ernst ne craignait rien, grâce à son costume, et surtout grâce à ce masque merveilleux de la misère qui lui ouvrait toutes les portes de l'ombre et, au-delà de la nuit, toutes les portes de l'enfer ${ }^{19}$.

Il ne s'agit pas là d'une plate idéalisation de la pauvreté - mais d'une introduction à la complexité des choses. Aliénante et souvent représentée comme telle dans le roman de Mac Orlan, la misère n'en est pas moins une puissance qui, comme tout ce qui agit, participe de la vie ; en cela, elle présente des aspects insoupçonnés, un envers inquiétant dont le misérable peut s'emparer : la misère n'interdit pas l'héroïsme. Dans Le Bal du Pont du Nord, le lecteur est invité à vivre dans un univers façonné par la mémoire, c'est-à-dire par la présence et le retrait de ce qu'il faut bien appeler le sublime historique :

À cette époque, il me semblait que j'étais le centre d'une vie puissante, un peu comme une pieuvre dont les tentacules de toute taille s'étendaient, suçaient et étreignaient plus loin que je ne le soupçonnais moi-même ${ }^{20}$.

Quand il s'inscrit dans l'histoire, le sublime en révèle la face obscure, sidérante, à jamais indéchiffrable. La « pieuvre », le bestiaire fantastique du roman, ne sont plus des métaphores ou des hyperboles, - ils traduisent avec exactitude ce qui fut ressenti ; de cette épreuve, le « lieu » porte trace comme s'il avait été l'agent énigmatique de la tragédie et continuait d'être le conservateur des énergies latentes et dangereuses qui persistent à agir sur les personnages :

Je pensais [...] que la grande digue de Zeebrugge étendait sa tentacule courbe sur tous les personnages que j'avais connus depuis mon arrivée sur le Zwin ensablée ${ }^{21}$.

Dans ce roman, le sublime historique et l'effroi qu'il suscite ne contribuent pas à l'effondrement du sujet ; ces expériences valorisées sont au contraire recherchées en raison même de leur caractère excessif. Le souvenir de la guerre, ce déchaînement incontrôlé des intensités, est patiemment entretenu, comme si l'humain ne valait que lorsqu'il tend vers l'inhumain. Il est significatif que la phrase « j'étais le centre d'une vie puissante, un peu comme une pieuvre » soit ambiguë. L'image de la pieuvre se rapporte-t-elle au « je » ou à la « vie puissante » qui l'étreint et le dépasse ? Le sujet se voue à ce qui le dépossède de lui-même, c'est-à-dire de ses limites $^{22}$. L'analogie avec la pieuvre exprime l'expérience de l'intensité quand elle a l'histoire

\footnotetext{
${ }^{16} B P N, 136$.

${ }^{17} B P N, 155$.

${ }^{18}$ Je cite, presque au hasard : « des documents photographiques d'une puissance évocatrice absolue » $(B P N, 106)$; « il était devenu d'une habileté surprenante » $(Q B, 7)$; « son instinct surprenant » $(Q B, 16)$; « des forces d'une puissance prodigieuse » $(Q B, 50)$; « une idée qui tout d'un coup lui parut éblouissante » $(Q B, 14)$; «l'épanouissement divin de dix heures de sommeil » $(Q B, 55)$. Nombre d'occurrences sont sans doute empreintes d'une ironie plus ou moins saillante, le romanesque prenant souvent plaisir à dénoncer ses propres excès ; ainsi, au moment où Michel Kraus met en scène son suicide, « dans une tragédie mi-sentimentale, mi-littéraire dont il devait être à la fois l'auteur et le héros », ses cheveux apparaissent " d'une coloration merveilleuse et romantique » $(Q B, 108)$.

${ }^{19} Q B, 99$.

$20 B P N, 108$.

${ }^{21} B P N, 96$. La répétition du mot « tentacule » construit une « métaphore obsédante ».

${ }^{22}$ Cette analyse ne prétend pas épuiser la manière dont le roman pense l'événement historique. Le texte offre en effet des représentations plus humanistes de la guerre : la grandeur de l'homme ne se révèle jamais mieux que lorsqu'il est le centre où convergent les violences conjuguées de la nature et de la machine : «La mer retrouvait ses armes naturelles, ses fureurs subites, le rythme de sa force. Point n'était besoin d'y laisser flotter des mines pour la rendre plus homicide. Dans sa parure de soie grise et d'argent fin, elle semblait prendre à sa charge des catastrophes futures sans le secours du génie humain. Quand cette eau méprisante le désirait, les grands croiseurs de bataille bondissaient sur ses flots comme des bouchons. Mais ils étaient
} 
pour objet, pour théâtre et pour enjeu. Ainsi se constitue un petit cercle d'initiés qui se devinent et se recherchent : " quand on possède le goût des gens exceptionnels, on finit toujours par en rencontrer partout ${ }^{23} \gg$, note placidement le narrateur de l'histoire ${ }^{24}$.

\section{II - Comparaisons intensives}

Dans cet univers romanesque, tout auréolé d'intensités, rien ne captive autant que le devenir des forces mises en jeu dans la rencontre entre l'homme et le monde, ou entre des éléments du monde. Ainsi cette phrase : « la chaleur du foyer agissait sur les vêtements du jeune homme qui se ratatinaient et s'appauvrissaient encore sous l'effet de la chaleur ${ }^{25} »$. Le déploiement de cette phrase semble épouser le phénomène qu'elle représente. La succession des verbes est révélatrice. Le premier verbe est transitif - « agissait sur »-, car la force du feu ne peut s'extérioriser qu'en cherchant un objet sur quoi s'exercer; les deux verbes suivants sont réfléchis - « se ratatinaient et s'appauvrissaient »-, car le vêtement, privé d'énergie, ne peut que subir : il incorpore une force étrangère qui l'altère. La phrase accompagne avec jubilation le devenir de la force agissante : l'adverbe « encore », la coordination des verbes, la précision presque redondante du complément « sous l'effet de la chaleur » sont autant de marques qui renchérissent sur l'activité du feu. Or cette activité est dangereuse, car elle peut détruire ce dont elle s'empare. De fait, le feu ne brûle que pour lui-même : il est l'origine et la finalité de son propre rayonnement, et c'est pourquoi le nom " chaleur » est répété aux extrémités de la phrase $^{26}$. Pour Mac Orlan, les substantifs sont des gisements d'intensités : une intensité est une qualité qui émane de l'objet et qui, souvent, l'identifie : chaleur du feu, décrépitude du vêtement. La phrase est l'enceinte où paraissent ces intensités ; les verbes président à leur déploiement, puis à leur affrontement. Une force, ce n'est donc qu'une intensité mise en mouvement. La phrase qui s'écoule baigne dans le même flux temporel que les phénomènes sensibles dont elle rend compte. Selon cet imaginaire, la phrase, par son organisation, reflète la circulation des forces du monde. Pour le montrer, je m'en tiendrais à l'étude d'une seule figure : la comparaison intensive.

Dans certaines comparaisons, le substantif qui forme le « comparant » apparaît comme le prototype de la qualité que veut exemplifier le locuteur. Ces comparaisons semblent nées de l'observation de la réalité familière. Mais l'expression de l'intensité peut aussi revêtir une coloration fantastique. Ainsi se révèlent les deux polarités de l'intensité : elle rassure quand elle est gage de plénitude sensorielle ; elle inquiète quand elle conduit les objets à s'émanciper de la tutelle humaine. Les comparaisons qui suivent sont énoncées soit par le narrateur, soit par les personnages de l'histoire. Tous ne parlent pas le même langage ; mais par le jeu de ces comparaisons, tous peuvent un instant avoir l'impression de se référer au même univers stable, là où se valident les analogies construites par le discours. Ainsi, Jan de Houcke est-il présenté comme « un homme vêtu de noir, correct comme un notaire ${ }^{27}$ »; « le notaire » est le prototype de la qualité visée par l'adjectif ; le prototype saisit l'état de « correction » dans sa plus grande

\footnotetext{
hérissés de canons fulgurants. Et mépris pour mépris, les hommes montraient encore l'attitude la plus noble entre l'artillerie et la mer également en folie » $(B P N, 103)$.

${ }^{23} B P N, 179$.

${ }^{24}$ Comme tous les rescapés des grandes aventures, les demi-soldes de la mémoire sont à la fois « sublimes et ridicules », ou « sublimes et vaguement ridicules ». Ces épithètes caractérisent respectivement le « Cygne » et les « Aveugles » de Baudelaire, grand poète (après Vigny) de la mélancolie historique. Le héros baudelairien est "sublime » puisque, en esprit, il participe à la valeur, et « ridicule », parce que la valeur le condamne à l'impuissance dans un univers où la valeur s'est retirée. Ce type d'imaginaire irrigue le très beau roman historique de Sarah Vajda, Amnésie, Monaco, éd. du Rocher, 2006.

${ }^{25} Q B, 19$.

${ }^{26}$ Ce procédé porte en rhétorique le doux nom d'épanadiplose.

${ }^{27} B P N, 49$.
} 
intensité. Seppen vante « des moules aussi grasses que des poulets ${ }^{28} »$. Comparé et comparant appartiennent au même genre, mais ce n'est pas toujours le cas : « votre voiture [...] est propre et vernie comme un hanneton ${ }^{29}$ », estime Seppen. Gibson décrit un homme " haut comme une armoire et gracieux comme un bœuf embourbé ${ }^{30} »$. S'incarnant dans la voie de Gibson, le langage populaire donne libre cours à sa créativité : la coordination signale le parallélisme, qui fait valoir l'inventivité du locuteur. " Gracieux » est employé par antiphrase ; c'est le comparant qui permet de déchiffrer le sens de l'expression. Des rapports de contiguïté viennent resserrer les liens entre les deux termes de la comparaison : Seppen, qui est marin, " fumait comme un $\operatorname{cargo}^{31} »$. La fiction se déroule au bord de mer; le monde maritime est donc grand pourvoyeur d'images. Dans ce contexte, il n'est pas invraisemblable qu'un sous-marin soit « vif comme un congre $^{32}$ » et un rafiot " aussi noir qu'un requin ${ }^{33}$ ». De même, "Gibson, l'hôtelier était appétissant et honnête comme un grand jambon ${ }^{34}$ "; c'est là sans doute le type de jambon que l'hôtelier sert à ses clients. La coprésence des éléments dans le même champ référentiel apporte un surcroît de légitimité à la comparaison, en vertu du vieux proverbe selon lequel ce « qui se ressemble s'assemble ». Le jeu langagier reçoit l'aval du réel, à moins qu'il ne le configure, comme dans cette superbe image : " il se fuitait par la rue Girardon comme un courant d'air... ${ }^{35} »$. On imagine le personnage devenu l'un de ces courants d'air qui traversent la rue.

Quand les comparaisons sont introduites par un adjectif, le substantif qui sert d'étalon (ou comparant) apparaît comme porteur d'une intensité qualitative qu'il confère à l'élément qui lui est associé (le comparé); ainsi la figure construit-elle l'image d'un univers harmonieux, où tout élément tend à réaliser la plénitude incarnée par le prototype, sans se confondre avec lui pour cela. Mais quand la comparaison dépend d'un verbe, il en va tout autrement : "Rabe s'imbibait comme une éponge et se gonflait d'anéantissement créé par le lit ${ }^{36} » ;$ « [...] une marche militaire $[. .$.$] résonnait dans sa tête comme dans un microphone { }^{37} »$. Dans ces analogies, les termes ne relèvent pas du même genre ; la relation de ressemblance n'est pas étayée par la coprésence référentielle du comparé et du comparant. Entre un être humain et une éponge, que peut-il y avoir de commun ? Rien. Le procès « s'imbibait » ne peut affecter l'éponge comme il affecte l'homme. Le microphone peut-il être le prototype d'un corps humain ? Assurément non, et l'expression de l'intensité passe par une autre opération de pensée, qu'il convient de décrire. Dans la comparaison, la différence entre les deux éléments est acceptée comme allant de soi ; elle est située à l'arrière-plan ; au premier plan, en position saillante, se tient la ressemblance, dont l'affirmation motive la comparaison. Dans les comparaisons que nous étudions, la différence, en raison de son caractère problématique, fait obstacle à la saisie de la ressemblance. Pour résoudre cette tension interprétative, le lecteur est obligé de surenchérir sur la ressemblance et de la pousser jusqu'à l'identité ; ainsi surgit un autre univers que celui qui sert de référence. Dans ce monde nouveau institué par la figure, de nouvelles intensités ont cours ; et, de fait, Rabe s'imbibe tellement de ce qu'il ressent, son corps devient si réceptif, que la différence qui le sépare de l'éponge ou du microphone s'efface dangereusement...

Ainsi le lecteur entre-t-il de plain-pied dans un univers fantastique. La comparaison dissipe les apparences rassurantes ; elle révèle la présence de forces hostiles, prêtes à bondir : «Il dormait, affalé ainsi qu'un fusillés ${ }^{38}$ ». Entre un homme qui dort et un fusillé, la pensée

\footnotetext{
${ }^{28} B P N, 112$.

${ }^{29} B P N, 45$.

${ }^{30} B P N, 114$.

${ }^{31} B P N, 171$.

${ }^{32} B P N, 37$.

${ }^{33} B P N, 81$

${ }^{34} B P N, 57$.

${ }^{35} Q B, 86$

${ }^{36} Q B, 8$.

${ }^{37} Q B, 15$

${ }^{38} Q B, 59$
} 
réserve un abîme que la comparaison abolit ; cette abolition inquiète, parce qu'elle fait surgir un danger imprévu. « Un hôtel voisin [...] s'ouvrit devant lui telle une prison tenue par un bougnat $^{39} \gg$. Dans ces existences précaires, tout refuge abrite la possibilité d'une menace. Ou encore : « il fermait les yeux avec rage, comme on serre les poings dans la colère ${ }^{40}$ ». L'intensité se manifeste par une disproportion : rapportée à l'acte de fermer les yeux, la puissance musculaire mobilisée par le geste de serrer ses poings ne peut manquer, semble-t-il, de produire un effet destructeur. Parfois, la comparaison requiert un laborieux travail d'interprétation, car les repères familiers qui organisent la perception ordinaire s'estompent : « sa propre misère [...] le suivait partout comme une compagne dévouée ${ }^{41} » ;$ « [...] une capitale où tous mes désirs m'attendaient comme les enfants des écoles prêts à chanter une cantate ${ }^{42} »$. La comparaison heurte comme un paradoxe. Qu'est-ce que cette misère qui, allégoriquement, apparaît sous les traits d'une « compagne dévouée »? Je ne suis pas prêt à réduire la fracture interprétative en invoquant les bons secours de l'antiphrase : « dévouée » serait une ironie. N'est-il pas proposé, au contraire, de penser la misère qui avilit par la notion ô combien déroutante d'une persistance presque rassurante, la constance du malheur finissant par engendrer la constance de celui qui le vit, constance qui n'est pas la résignation d'une victime mais l'acceptation héroïquement joyeuse d'une vie incompréhensible ? Et quels sont ces « désirs » qui ont l'aspect d' " enfants » sages ? La misère ou le désir sont des puissances intenses qui bouleversent toutes les représentations : celles des personnages comme des lecteurs. Ces comparaisons s'engrènent sur des métaphores, portées par les verbes : la misère « suit » le misérable, les désirs « attendent » docilement l'homme qui les éprouve. La métaphore verbale donne à des termes abstraits ( « désirs », « misère ») une intention ou une psychologie humaines qui préparent la comparaison anthropomorphique ; mais au lieu que le mouvement allégorique ainsi engagé soit confirmé par une analogie acceptable, c'est une image décalée qui s'impose. L'intensité cesse d'être cette vertu cohésive qui unifie l'univers; elle le dérègle ou le délabre. Si l'intensité est une énergie à ce point ambivalente, comment l'homme doit-il se situer par rapport à elle ? Les récits de Mac Orlan ont sans doute beaucoup à nous apprendre à ce sujet.

\section{III- Intensité et subjectivité}

Chaque phrase de Mac Orlan semble pouvoir être décrite comme un dispositif intensif. Si l'on en croit Gorgio Agamben ${ }^{43}$, qui a consacré un petit texte à cette notion, un dispositif est un ensemble hétérogène de règles, de rites, de lois, qu'un pouvoir extérieur impose à l'individu et que celui-ci intériorise dans un système de croyances et de sentiments. Agamben précise : « le terme dispositif nomme ce en quoi et ce par quoi se réalise une pure activité de gouvernement sans le moindre fondement dans l'être. C'est pourquoi les dispositifs doivent toujours impliquer un processus de subjectivation. Ils doivent produire leur sujet ${ }^{44} \gg$. Risquons le geste sacrilège qui consiste à dépolitiser la notion, à la déporter de son champ originel (l'analyse du pouvoir) pour l'éprouver au contact d'un texte littéraire. Chaque phrase où s'actualise le « réseau stylistique » de l'intensité constitue un dispositif en ce qu'elle construit le sujet (fictif) qui perçoit cette intensité et qui en est affecté. Mieux : la phrase de Mac Orlan évalue ces processus de subjectivation : elle juge la manière dont le sujet réagit à cette intensité ; mais, contrairement aux « activités de gouvernement», rien ne permet de dire que ces dispositifs soient dénués du «moindre fondement dans l'être ».

\footnotetext{
${ }^{39} Q B, 9$.

${ }^{40} Q B, 110$.

${ }^{41} Q B, 11$.

${ }^{42} Q B, 52$.

43 Gorgio Agamben, Qu'est-ce qu'un dispositif ?, traduit de l'italien par Martin Rueff, Paris, éd. Payot et Rivages, coll. «Rivages poches », 2007. Je remercie Grégory Berrué d'avoir attiré mon attention sur ce texte.

${ }^{44}$ Qu'est-ce qu'un dispositif?, ouvrage cité, pp. 26-27.
} 
Lisons cette phrase : « le carillon s'empara de la ville, la martela, quart d'heure par quart d'heure, gentiment mais inexorablement ${ }^{45}$ ». Le texte pose l'existence d'un dispositif intensif. Il est lié à la métaphore agentive que porte la construction verbale. À l'origine de la propagation du son, il y a une initiative : « le carillon s'empara de la ville, la martela ». Cette initiative ne peut manquer d'impliquer un sujet; celui-ci est affecté par la sensation et capable d'évaluer son intensité : «gentiment mais inexorablement ». À quoi tient la gentillesse prêtée au carillon ? À ceci que l'intensité perçue ne cherche pas à nuire au sujet sur lequel elle étend son emprise. Sans ce geste inaugural de captation, il n'y a pas l'intensité : le sujet n'est pas libre de refuser ou d'accepter la rencontre avec l'intensité. C'est en cela qu'il est sujet : il est soumis à ce qui le sollicite. Tout cela, Mac Orlan le reconnaît volontiers, n'est qu'un imaginaire : «j'ai tenté de réunir les images d'un romantisme adapté à mon inquiétude : Psychologie du décor qui domine la psychologie de l'homme ${ }^{46} \gg$.

Le sens des mots importe. Une psychologie est un logos qui se rend attentif à la réalité de l'âme. Le carillon s'entend. Autour de ce mot (ou de cette sensation) se déploie tout un décor (urbain, en l'occurrence). Mais l'homme qui entend le carillon, lui, ne s'entend pas lui-même alors qu'il perçoit ce qui vient frapper son oreille. Il est une âme imperceptible quoique immanente et jointe au corps ; c'est à cette réalité de l'âme que se voue le romancier psychologue. Cette âme ne vit que par l'action qui la révèle à elle-même : c'est ce qu'indique le verbe « domine ». On cerne le cœur du dispositif intensif. Mac Orlan s'intéresse aux âmes qui perçoivent la vie comme un champ d'intensités en perpétuelle évolution ; ces intensités nous sont tour à tour favorables ou hostiles; elles augmentent en nous la vie, ou la détruisent. On ne sait si elles sont dotées d'une intention ou si leur action s'exerce avec indifférence sur les sujets dont elles mettent l'âme à nu. Cette indécision définit exactement ce que Mac Orlan nomme le mystère : une femme déterminée, dit-il, « est une force de la nature comparable à l'électricité dont on asservit les caprices sans rien pénétrer de son mystère originel ${ }^{47}$ ». Les héros de Mac Orlan ne cessent d'être aux prises avec ces intensités qui, en les croisant, engendrent en eux non une nature stable, mais des subjectivités successives, passagères, dont le flux continuel est celui de la vie. Gibson peut en témoigner :

Dès les premiers jours du mois, je pense à la nuit du 22 avril 1918. Alors tout ce que je touche me plaît, tout ce que je vois m'émerveille et la plus rude des nuits au bord de la mer me paraît douce comme une couverture ${ }^{48}$.

Gibson se sait partie prenante d'un dispositif, qui est celui de l'intensité historique. Le «je » qui parle, qui sait si bien ce qu'il vit et qui sait si bien le dire, ne s'engendre pas lui-même ; à proprement parler, il n'est pas libre d'être ceci ou cela, ceci puis cela ; il n'y va pas, comme le dit si bien Rimbaud, de sa « faute ». Cela n'empêche nullement les êtres vivants de se comprendre, eux-mêmes et les autres, et de s'aimer.

Par vocation, la littérature participe à la grande œuvre du partage des intensité, et c'est en cela, par un biais inattendu, que l'imaginaire de Mac Orlan prend un tour politique :

[...] le livre de Charles de Coster rayonnait. Sa puissante lumière si douce et si vivante ne pouvait plus être remplacée par un autre miracle littéraire, si on admet que la littérature devient dans ce cas la forme la plus populaire d'un socialisme national presque divin ${ }^{49}$.

Élucubration ? Il me semble au contraire que chaque mot compte. Charles de Coster le prouve : il arrive que la littérature devienne elle-même intensité ; elle rayonne, se charge de produire des sujets - mais non pour les soustraire à l'être, mais pour les plonger dans une vie collective

\footnotetext{
${ }^{45} B P N, 162$.

${ }^{46} B P N, 18$

${ }^{47} Q B, 131$.

${ }^{48} B P N, 30$.

${ }^{49} B P N, 36$.
} 
renouvelée. « Socialisme » signifie partage : il n'y a aucune raison d'exclure ceux qui veulent participer à l'aventure ${ }^{50}$. La richesse commune est indivise : il en va d'elle comme de l'amour maternel, « chacun en a sa part, et tous l'ont tout entier ». C'est cette égalité utopique que réalise le livre inspiré, celui qui parle à tous, et de chacun. Ce socialisme est national, parce qu'il s'ancre dans un lieu précis, qui confère une identité politique à ceux qui y vivent. La littérature des intensités est inspirée par un « décor », cet espace concret à la puissance duquel on ne peut que se soumettre. Ce socialisme est " presque divin », parce qu'il se nourrit de mystère, décrit des présences qu'on éprouve sans les comprendre, et dont l'emprise surréelle donne l'impression de basculer dans l'irréalité :

Tous les Allemands toussotaient en portant les mains à leur cou, car ils croyaient toujours sentir le frôlement des doigts d'un étrangleur illuminé par l'amour de la Flandre libre $^{51}$.

L'envahisseur allemand fait à ses dépens l'expérience de l'intensité : en lui, la sensation du contact se dépasse elle-même dans l'hallucination d'une présence presque folle, passionnée, la présence d'un « étrangleur illuminé par l'amour ». Le mot clé nous est livré : l'amour. Et au regard de l'intensité où il nous implique, l'objet de cet amour - la « Flandre libre », ou de quoi ce soit d'autre - importe somme toute assez peu.

\footnotetext{
${ }^{50}$ À Bruges, le narrateur acquiert « la certitude » de n'être jamais qu'un « étranger » dans la foule « joviale et réservée » qui l'entoure $(B P N, 46)$. Soit. Mais il n'en est pas moins invité à commémorer l'épopée flamande, dont la mémoire se perpétue autour de la table, où il n'y a que des égaux : «Comme tous mes compagnons, j'avais vite pris l'habitude de ne parler qu'à mon tour. L'idée tournait d'elle-même comme un toton. Elle s'arrêtait tout net, au hasard, devant l'un de nous. C'était à cet homme de parler » $(B P N, 41)$

${ }^{51} B P N, 51$.
} 